\title{
ACDV, Association Chimie du Végétal : une initiative française pour développer la chimie du végétal
}

\author{
Aurore FRIÈS \\ UIC, \\ Union des industries chimiques, \\ Le Diamant $A$, \\ 92909 Paris La Défense Cedex, \\ France \\ $<$ afries@uic.fr>
}

Article reçu le 13 novembre 2012

Accepté le 10 décembre 2012

\begin{abstract}
A novel initiative towards developing biobased chemistry for a sustainable future: $A C D V$, the French biobased chemistry association

Industry dependence on external fossil fuel resources, fluctuations in energy and raw materials costs, climate change, environmental regulations, changing consumer attitudes etc. In France, where agriculture and the chemical industry are two pillars of the economy, these harsh realities have prompted the chemical and agro-resources sectors to look to biobased chemistry as a means of diversifying outlets for biomass and chemical sourcing, with the aim of balanced use. French players in this international sector have realised the value of joining forces, pooling skills, knowhow and resources into a single structure to drive forward the development of biobased chemistry. This shared vision and determination resulted in the creation in 2008 of the French biobased chemistry association, Association Chimie du Végétal (ACDV). This initiative, still the first of its kind, brings together within a single federation all those directly involved in biobased chemistry. ACDV's stated aim: to propose and create industrial, economic and political conditions conducive to the development of this industrial reality, within a context of competitive, responsible and sustainable agricultural and chemical sectors. $A C D V$ acts as a source of creative ideas, presenting national and European bodies with the strategic vision and position of French industrialists as regards the international development and competitiveness of biobased chemistry. A commitment by the French chemicals industry to a target of $15 \%$ biobased raw materials by 2017 is one of the key factors underpinning the work of the ACDV.
\end{abstract}

Key words: agro-resources, biobased chemistry, networking association, ACDV

L'indicateur du contenu biosourcé, la méthode d'analyse de cycle de vie spécifique à la chimie du végétal ou encore les colloques pour les acteurs du développement sont autant d'exemples de la volonté de faire avancer la chimie du végétal. Au vu du cœur de métier de ses membres, chimistes et bioraffineurs pour la plupart, et de ses champs d'actions, promotions, études et analyses, consultations, I'ACDV est un acteur-clé pour représenter la chimie du végétal tant au niveau français qu'au niveau européen.

Notre société est en constante évolution, elle découvre de nouveaux procédés, elle invente de nouvelles technologies, elle change ses comportements de consommation, elle se soucie d'avantage de son environnement. Tous ces changements se font sans que l'on s'en aperçoive mais ils impactent directement notre quotidien. La chimie du végétal est I'un de ces changements. Elle a naturellement découlé des innovations industrielles de ces dernières années tout en répondant aux attentes de notre société en pleine mutation. La chimie du végétal offre une ressource complémentaire aux ressources fossiles dans l'industrie chimique. Elle permet ainsi de réduire notre dépendance aux importations.

De plus, depuis les années 1980, il est apparu que les ressources carbonées fossiles de la planète (charbon, pétrole, gaz naturel) ne suffiraient bientôt plus à couvrir les besoins croissants en énergie et en produits chimiques dus à la croissance démographique ainsi qu'à l'évolution globale du niveau et du mode de vie. D'autre part, fabriquées 
à partir de $\mathrm{CO}_{2}$ atmosphérique et utilisant des bioprocédés, la matière première biosourcée contribue à diminuer l'impact environnemental de I'industrie. La chimie du végétal répond à ce double défi et présente une alternative crédible afin de passer d'une économie du tout pétrole vers une utilisation croissante des ressources renouvelables, ainsi que sur une meilleure gestion et valorisation des ressources de la biomasse.

Pour répondre à ces défis, les industriels de la chimie s'engagent vers un objectif de $15 \%$ d'utilisation de ressources végétales en tant que matière première de la chimie d'ici à 2017'. L'avenir de la chimie du végétal se joue en ce moment à travers le monde. Pour que l'Europe et la France saisissent cette opportunité $d^{\prime}$ innovation et se posent en leaders mondiaux de ce secteur, les pouvoirs publics doivent dès aujourd'hui prendre conscience de l'importance de cette nouvelle filière et soutenir son développement.

\section{Les forces $d$ 'une filière avec des perspectives pour I'avenir}

Les membres de I'ACDV qui se lancent dans ce secteur prometteur peuvent s'appuyer sur des avantages compétitifs existants : des sites industriels pérennes, des centres de recherches innovants, des matières premières suffisantes ou encore une chaîne logistique rodée. Mais, malgré ces avantages, les acteurs des univers de la chimie et des agroindustries ainsi que les industries utilisatrices finales ont pris conscience que la mutualisation de leurs forces servirait un développement plus rapide de la chimie du végétal en Europe.

Le développement de la chimie du végétal se construit sur une base économique et industrielle forte :

- Pour l'industrie chimique (UIC, 2011) : - I'Europe est au deuxième rang mondial, et la France est le $5^{\mathrm{e}}$ pays producteur,

- en France, la chimie pèse 86,7 milliards d'euros de chiffres d'affaires soit $1 \%$ du PIB français,

- et représente 156500 emplois directs ;

\footnotetext{
${ }^{1}$ Objectif proposé par les industriels de la chimie lors du Grenelle de I'environnement.
}

- Pour le secteur agricole :

- la France est le premier pays agricole de I'UE, et se positionne au $1^{\mathrm{er}}$ rang européen pour la production de céréales et d'oléagineux,

- en 2008, le secteur agricole et agroalimentaire représentait 66,8 milliards d'euros soit 3,5\% du PIB, et 770000 emplois (chiffres 2007).

Les matières premières représentent un enjeu majeur pour le développement de la chimie du végétal qui se définit par la nature renouvelable des matières premières qu'elle transforme. Les capacités de productions agricoles françaises et européennes sont aujourd'hui estimées par les acteurs du secteur, comme suffisantes pour pouvoir répondre à ces nouvelles exigences.

Par leur position de leader agricole, la France et I'Union européenne sont à même de fournir aux industriels des matières premières en quantité et qualité mais également à des prix compétitifs. En effet, si l'on considère l'approvisionnement pour la filière chimie du végétal du point de vue de I'utilisation des surfaces mobilisées :

- I'Union européenne est à la $3^{\mathrm{e}}$ place mondiale en termes de production céréalière ;

- I'UE à 27 a produit, en 2008, 313 millions de tonnes de céréales sur 60 millions d'hectares, 27 millions de tonnes d'oléagineux sur 10 millions d'hectares et 17 millions de tonnes de sucre ; - la surface forestière de l'UE à 27 est de près de 160 millions d'hectares (Mha) ; - de plus, I'Union européenne a l'avantage d'avoir des filières d'approvisionnement agro-industrielles et de la forêt bois structurées et pleinement opérationnelles.

Ainsi, à I'horizon 2020-2025 la consommation de matières premières carbonées de la chimie européenne représenterait $82,5 \mathrm{Mt} / \mathrm{an}$ de carbone dont environ $16 \%$ pourraient être issus de la biomasse (12,5 Mt de carbone renouvelable) (source : PIPAME, 2010).

Si une estimation simple ${ }^{2}$ est faite de la surface requise pour produire les 12,5

\footnotetext{
${ }^{2}$ Estimation : rendement éthanol 4-7 t/ha (selon biomasse et conversion utilisée) soit 23 t carbone/ha, donc si les 12,5 Mt de carbone nécessaire pour la chimie provenaient entièrement de l'éthanol, il faudrait 46 Mha de terres agricoles et forestières.
}

Mt de carbone estimées nécessaires en 2020-2025, avec les différentes ressources de la biomasse actuelle (grandes cultures, résidus lignocellulosiques de céréales, et bois), cela équivaudrait à utiliser 4 à 6 Mha, soit environ 2-3\% des surfaces agricoles et forestières européennes. Par comparaison, avant 2009, les jachères obligatoires prévues par la PAC étaient d'environ 4 Mha soit $8 \%$ des terres agricoles européennes.

De plus, le maillage agricole français ainsi que l'implantation régionale des filières favoriseront le développement économique de la filière du végétal mais apporteront également de nouvelles perspectives pour ces régions et acteurs :

- création d'emplois dans les régions ;

- nouveaux débouchés agricoles;

- pérennisations d'outils industriels ;

- dynamique économique régionale.

La pérennisation des implantations agricoles en France et en UE est également une des perspectives de la chimie du végétal. En effet, celle-ci permettra aux agriculteurs d'envisager de nouveaux débouchés pour leur production dans un contexte économique ou la tendance va vers une revente des terres agricoles pour les besoins de l'urbanisation ${ }^{3}$. La complémentarité des acteurs aujourd'hui engagés dans la chimie du végétal, les avantages existants et futurs en termes d'approvisionnement ainsi qu'une volonté de créer de la richesse, font de la France un chef de file désigné et légitime pour se positionner auprès des institutions françaises et européennes.

\section{Des enjeux stratégiques}

"L'industrie chimique est aujourd'hui fortement dépendante des ressources fossiles dont elle tire la majorité de ses produits. La raréfaction de ces ressources, la variabilité de leur coût et les enjeux géopolitiques associés imposent de trouver des matieres premieres de substitution, renouvelables telles que la biomasse. La chimie du végétal a un rôle majeur à jouer pour contribuer à réduire cette dépendance et d'autre part dans le

\footnotetext{
${ }^{3} 19,5$ millions d'hectares de terres agricoles sont converties chaque année en terres pour le développement industriel et immobilier selon un rapport de l'ONU d'août 2010.
} 
développement de produits plus respectueux de la santé et de l'environnement. " Cet extrait de la feuille de route ADEME sur la chimie du végétal illustre en quoi la chimie du végétal a un rôle important à jouer dans les politiques de durabilité de la France et d'indépendance énergétique et industrielle de l'Europe pour les 20 prochaines années. En effet, le développement durable regroupe trois aspects : économique, environnemental et sociétal auxquels la chimie du végétal est à même de répondre. Pour l'environnement, les procédés mis au point en chimie du végétal doivent répondre à un objectif d'économie d'énergies, d'eau et de solvants, de limitation des rejets et d'amélioration des rendements, conformément aux principes de la chimie durable.

De plus, en termes d'approvisionnement de matières premières, un travail en collaboration avec les secteurs agricoles et forestiers sera nécessaire pour s'assurer de la qualité de la biomasse mais aussi pour garantir une biomasse cultivée en conformité avec les critères de durabilité (conforme à la législation européenne existante).

Répondre aux enjeux économiques est également une des priorités dans laquelle s'engage la chimie du végétal. Aussi, le développement économique de la filière chimie du végétal nécessite de :

- développer des filières industrielles compétitives ;

- offrir de manière régulière des produits en quantité et qualité satisfaisantes et à un niveau de prix de compétitivité mondiale compte tenu des propriétés apportées ;

- développer les emplois directs et induits (industriels et agricoles).

Le développement économique de la filière chimie du végétal est influencé par le choix des filières porteuses, la compétition entre filières, le développement de modèles économiques et l'accès aux marchés.

À ces paramètres économiques, il faut enfin ajouter les aspects sociétaux. Ainsi, depuis une dizaine d'années, le consommateur mais aussi les entreprises utilisatrices finales demandent des produits du quotidien plus verts, moins impactant sur l'environnement. La chimie du végétal doit également répondre aux challenges de demain que sera une population plus importante avec des habitudes de consommation qui vont évoluer vers une demande plus forte en biens de consommation. Le défi sera pour les industriels de I'ACDV de répondre à ces besoins sans impacter la chaîne de production alimentaire. En effet, la chimie du végétal ne peut se développer que dans le cadre d'un équilibre global avec les utilisations traditionnelles des ressources végétales, en premier lieu l'alimentation humaine et animale.

Pour assurer cet équilibre, plusieurs voies de développement coexistent :

- la valorisation alimentaire et non alimentaire de l'ensemble d'une plante au travers des bioraffineries: I'idée est de faire coexister les usages alimentaires (humains, animaux) et non alimentaires dans une même unité de production pour exploiter tout le potentiel de la biomasse ;

- I'utilisation de ressources non alimentaires telles que le ricin, le bois, avec I'utilisation pour la chimie de coproduits issus de l'industrie papetière ou encore à l'avenir les micro-algues, dont le rendement potentiel est très élevé ;

- le développement de la capacité à utiliser des résidus de la biomasse : plusieurs projets de R\&D sont ainsi engagés pour exploiter les parties non alimentaires d'une plante, notamment la cellulose extraite de la tige des plantes.

Cet équilibre est rendu possible par les bioraffineries, un outil industriel qui, sur un même site, isole, traite et valorise chacun des composants de la biomasse, chaque constituant de la biomasse est séparé et valorisé en aval.

\section{Des opportunités à saisir.. .}

Selon le cabinet de consultants Arthur D. Little (Palastro, 2012), le marché des produits chimiques biosourcés représente actuellement au niveau mondial entre 50 et 70 milliards d'euros soit 3 à $4 \%$ des ventes actuelles de la chimie en incluant la chimie fine pharmaceutique. À I'horizon 2025, cette même étude évalue le marché mondial des bioproduits entre 175 et 420 milliards d'euros soit entre 7 et $17 \%$ du marché mondial de la chimie. D'autres estimations telles que celles de McKinsey (CEFIC, 2011) prévoient un marché de l'ordre de 220 à 240 milliards d'euros en 2020 pour les produits chimiques biosourcés.

Même si beaucoup d'incertitudes existent encore quant au développement massif à I'horizon de 10-15 ans de la chimie des produits biosourcés, on peut d'ores et déjà être confiant grâce aux réalisations des industries du secteur, par les pôles de compétitivité et I'ACDV.

Cet engagement permet ainsi de pouvoir prédire pour les années à venir des taux de pénétration de la chimie du végétal satisfaisants compte tenu de la nouveauté des technologies. Ainsi, en France, I'ACDV estime que le taux de pénétration de la chimie végétale au niveau mondial à horizon 2025 devrait être d'environ :

- $20 \%$ des polymères, répartis à $80 \%$ pour les polymères de spécialités et à $20 \%$ pour les polymères de base (38 Mt de carbone par an);

- 5 à $6 \%$ pour les intermédiaires de commodité hors polymères (6,6 Mt de carbone par an);

- $20 \%$ de la chimie organique pour les produits de spécialités et de chimie fine (20 Mt de carbone par an).

Soit au total un taux de pénétration mondial d'environ $16 \%$ de produits biosourcés en chimie à l'horizon 2025 (sur la base des 400 Mt de carbone utilisées aujourd'hui par la chimie mondiale).

La France est bien placée pour être I'un des principaux producteurs européens de produits biosourcés grâce à des matières premières disponibles en qualité et quantité suffisantes pour répondre aux futurs besoins de la chimie du végétal ou bien grâce à des acteurs déjà implantés dans ce secteur hautement compétitif au niveau mondial. Ce développement agricole et industriel a pour ambition d'être réalisé en France ainsi qu'en Europe en s'appuyant sur des atouts déjà solides.

Une chimie du végétal française ne pourra se faire qu'à partir d'un secteur agricole fort, qui saura lui aussi exploiter les bénéfices de cette nouvelle filière du végétal d'un point de vue économique, social et environnemental.

Cependant ce développement ne se fera qu'à condition que les industriels 
des agro-industries continuent à renforcer leur compétitivité dans les filières actuelles, en particulier avec des bioraffineries intégrées et dans les filières futures avec la biomasse de seconde (bois...) et troisième générations (algues...).

L'ensemble des acteurs réunis autour des nombreux projets ${ }^{4}$ réalisés ou en cours de réalisation tendent à démontrer que la France doit continuer de proposer des solutions innovantes et d'encourager, via différents systèmes, la prise de risque que représente le secteur des produits biosourcés. Les programmes initiés par le Gouvernement français : Grand emprunt (PIVERT ${ }^{5}$ (Rous, 2012), Indeed ${ }^{6}$ et IFMAS ${ }^{7}$ pour ne citer qu'eux), comités consultatifs, ou I'Europe : LMI $^{8}$, Star Colibri commencent à peine à porter leurs fruits et il est donc important de continuer dans cette trajectoire.

Cependant, afin de permettre un développement et une intégration plus poussée de la filière, certains verrous organisationnels doivent encore être levés.

\section{...malgré des améliorations à apporter, des problèmes identifiés}

Le développement de la chimie du végétal est aujourd'hui restreint par certaines difficultés identifiées ci-dessous :

\section{La compétitivité internationale de nos entreprises}

La filière des matériaux biosourcés est aujourd'hui directement concernée par la compétition mondiale face à des acteurs comme les États-Unis, le Brésil et certains pays d'Asie qui ont pris tôt conscience du potentiel de ce nouveau

\footnotetext{
${ }^{4}$ La liste des réalisations industrielles des membres de l'ACDV est disponible auprès de I'ACDV.

${ }^{5}$ Picardie Innovations Végétales Enseignements et Recherches Technologiques.

${ }^{6}$ Institut national pour le développement des écotechnologies et des énergies décarbonées.

${ }^{7}$ Institut Français des Matériaux AgroSourcés.

${ }^{8}$ Lead Market Initiative.
}

secteur et soutiennent son développement sur leurs sols.

Cette compétitivité passera par un niveau de jeu égal avec nos compétiteurs internationaux. De nombreux pays mettent en place des régimes de prêts ou de subventions pour attirer les investissements étrangers. C'est particulièrement le cas en Asie ou aux États-Unis où de nombreuses technologies innovantes y compris européennes commencent à y être industrialisées.

\section{Des verrous réglementaires}

Pour ne pas perdre notre avance européenne, il est important de traiter dès aujourd'hui les verrous qui bloquent ces développements. Le poids toujours plus important des réglementations européennes et françaises qui ne sont parfois pas coordonnées, augmente la lourdeur du cadre d'évolution (exemple : REACH).

Ces réglementations sont parfois plus importantes et drastiques en Europe que dans le reste du monde. Elles apportent des contraintes supplémentaires aux entreprises européennes qui ont déjà du mal à être compétitives économiquement. II faut éviter de créer des distorsions de concurrence liées à la règlementation.

\section{Un manque de cohérence et d'harmonisation}

Le développement de la filière chimie du végétal en Europe ne fait pas l'objet d'un cadre politique européen clair et harmonisé. Ceci est d'autant plus important que la chimie du végétal touche à de nombreux secteurs économiques et enjeux sociétaux tels que la sécurité alimentaire, la sécurité et I'indépendance énergétiques, la lutte contre le réchauffement climatique, le développement des ressources renouvelables, le développement agricole et des zones rurales. Actuellement, I'Europe et les États membres mettent en place un certain nombre de programmes pour proposer des solutions à ces enjeux mais sans coordination transversale (entre les différents enjeux) ou verticale (entre I'UE et les États membres) sur le développement et la mise en action de ces programmes. II en résulte un manque de clarté et de visibilité sur le long terme de la politique européenne dans ce secteur, une fragmentation des programmes existants et un manque de cohérence des mesures mises en place.

\section{En conclusion}

L'ACDV envisage deux recommandations pour soutenir le développement de la chimie du végétal en France et en Europe :

1) une harmonisation et une coordination des politiques de développement de la chimie du végétal :

- mise en œuvre des recommandations du Lead Market Initiative pour les produits biosourcés,

- établissement d'une plateforme interministérielle française simple et efficace pour établir une stratégie commune et son déploiement de manière coordonnée ;

2) une stratégie forte pour le soutien au développement économique de la filière :

- une fiscalité avantageuse pour les produits biosourcés : crédit d'impôt accordé sur la base du contenu végétal de certains intermédiaires ou produits finis, TVA réduite, faciliter le crédit pour les produits biosourcés...,

- la définition d'objectifs chiffrés, ce qui permettrait d'accompagner et de vérifier l'impact des mesures mises en œuvre,

- I'introduction d'une préférence aux produits biosourcés dans les achats publics pour une liste déterminée de produits,

- la mise en place d'une labellisation permettant de valoriser les bénéfices environnementaux des produits biosourcés et de visualiser le contenu en matières biosourcées sur les emballages des produits de grande consommation.

Conflits d'intérêts : non déclarés.

\section{RÉFÉRENCES}

CEFIC. Future of chemical industry, vision 2030. Mc Kinsey. Confidential presentation. 2011.

Polastro E. La chimie du végétal en France et dans le monde à l'horizon 2020. Conférence organisée par l'Usine Nouvelle - Chimie du 
végétal, Bioraffineries: vers une troisième révolution industrielle. 15 mars 2012. Présentation téléchargeable sur: http:// www. usinenouvelle.com/article/conference-chimie-du-vegetal-15-mars-2012. N171034 (dernière consultation le 26/11/ 2012).
Pôle interministériel de prospective et d'anticipation des mutations économiques, PIPAME. Mutations économiques dans le domaine de la Chimie. Février 2010. 141 pages. Consultable sur: http://www.industrie.gouv.fr/ p3e/etudes/chimie/chimie_abrege.php (dernière consultation le 26/11/2012).
Rous JF. Le projet français P.I.V.E.R.T. OCL $2012 ; 19: 370-8$.

UIC. L'industrie Chimique en France Chiffres et idées clés. Mars 2012. Consultable sur http://www.uic.fr/edition-34433-chimiebref-chiffres-idees-chimie-france (dernière consultation le 26/11/2012). 